\section{Mediterranean Diet Food: Strategies to Preserve a Healthy Tradition}

\author{
Dimitrios Boskou* \\ Aristotle University of Thessaloniki, Greece
}

"Corresponding author: Dimitrios Boskou, Aristotle University of Thessaloniki, Greece, Tel: +30 2310997791; E-mail: boskou@chem.auth.gr

Received date: September 21, 2015; Accepted date: November 28, 2015; Published date: December 7, 2015

Copyright: (c) 2015 Boskou D. This is an open-access article distributed under the terms of the Creative Commons Attribution License, which permits unrestricted use, distribution, and reproduction in any medium, provided the original author and source are credited.

\begin{abstract}
The traditional Mediterranean diet refers to a dietary pattern found in olive growing areas of the Mediterranean region. It's essential characteristic is the consumption of virgin olive oil, vegetables, fresh fruits, grains, pasta, bread, olives, pulses, nuts and seeds. Moderate amounts of fish, poultry, dairy products and eggs are consumed with small amounts of red meat and wine. Over the past few decades there has been a growing interest in the role of the Mediterranean diet in preventing the development of certain diseases, especially cardiovascular disease. Mediterranean food products are now re-evaluated for the beneficial health effects in relation to the presence of bioactive compounds. The body of science unraveling the role of bioactives such as phenolic acids, various polyphenols, flavonoids, lignans, hydroxyl-isochromans, olive oil secoiridoids, triterpene acids and triterpene alcohols, squalene, alpha-tocopherol and many others is growing rapidly. A challenge for future research is the magnitude of the contribution of each active compound to the overall positive health effect. Strategies to preserve and disseminate the healthy Mediterranean diet should focus on: the implementation of the claim recently approved by EFSA for the level of biophenols in olive oil and the protection of LDL oxidation; technological improvements based on the increased awareness about the role of minor constituents of Mediterranean foods; products that are innovative but also traditional.
\end{abstract}

\section{The Traditional Mediterranean Diet}

The traditional Mediterranean diet refers to dietary patterns typical of specific regions of the Mediterranean region in the early 1960s. It is characterized by an abundance of plant foods such as vegetables, fresh fruits, grains, pasta, bread, pulses, nuts and seeds, and a high level of monounsaturated fatty acids. A common feature of the Mediterranean diet is a high consumption of olives and olive oil as the primary sources of dietary fat and moderate amounts of fish, poultry, dairy products and eggs consumed along with small amounts of red meat and wine.

Over the past few decades there has been a growing interest in the role of the Mediterranean diet in preventing the development of certain diseases, especially cardiovascular disease. The discovery of the cardioprotective properties of the diet is one of the great successes of epidemiology. Today there are many biochemical and other studies in the field of biosciences related to Mediterranean food products and their ingredients. These studies confirm the findings of epidemiology related to vascular health and hypertension and indicate also that a high intake of foods typical of the Mediterranean dietary pattern and a good adherence to it provides protection against coronary heart disease and may be inversely associated with the development of various types of cancer, arthritis, diabetes and neurodegenerative diseases. They may also be effective in improving health status and reduce mortality [1-3].Thus, the pattern has been garnering interest throughout the world and more people are interested in the health benefits it confers.

\section{Mediterranean diet pyramids}

The healthy traditional Mediterranean pattern has been represented by various pyramids indicating graphically the foods to be consumed on a daily basis or weekly. Mediterranean diet pyramids have been continuously re-designed and completed. The last 2010 version (Eighth International Congress on The Mediterranean Diet, Barcelona), incorporates lifestyle and cultural elements such as moderation, conviviality, seasonality and eco-friendliness. In other words, the new revised modern Mediterranean diet takes into consideration contemporary lifestyles and environmental constraints and is compatible with the development of a sustainable diet model for present and future generations [2].

\section{Mediterranean Food Products}

The great progress made in the areas of natural products chemistry, food analysis, nutrition, biochemistry and other biosciences during the last two or three decades has demonstrated that some commonly encountered food constituents have hitherto unknown health promoting and disease preventive properties. Mediterranean food products are now re-evaluated for the beneficial health effects in relation to the presence of bioactive compounds. A lot of information is now available. A good example is biophenols.

Phenolic compounds widely distributed in the plant kingdom and abundant in our diet are today among the most talked about classes of phytochemicals. This is indicated by the accumulated scientific work that focuses on:

- Oxidation mechanisms and contribution of phenols and generally natural antioxidants in preventing free radical damage and oxidative stress

- The dietary intake of phenolic compounds and its effect on lipoprotein metabolism, oxidative damage, inflammation, endothelial dysfunction, and blood pressure

- The potential of certain phenols in oncology, chemoprevention, cell-specific cytotoxic and apoptotic effects 
- The clarification of molecular mechanisms accounting for the antioxidant, anti-inflammatory, and anticancer properties through gene transcription profiling

- The extraction of phenols and other bioactive compounds from fruits processing residues

\section{Virgin olive oil}

Virgin olive oil is a fundamental Mediterranean diet component and contributes substantially to the health benefits of this diet. It is rich in mono-unsaturated fatty acids and contains bioactive phenols such as the dialdehydic forms of elenolic acid linked to tyrosol and hydroxytyrosol, oleuropein and ligstroside aglycons, tyrosol and hydroxytyrosol. Other polar phenolics present in virgin olive oil are lignans, phenolic acids, flavonoids, derivatives of phenolic alcohols, hydroxy-isochromans and traces of glycosides (oleuropein, ligstroside). Non polar bioactive constituents and other nutritionally important compounds are alpha-tocopherol and non-phenolic compounds, mainly squalene hydroxyterpenic acids, triterpene dialcohols, phytosterols and carotenoids [4].

The present knowledge of olive oil's complex composition is due to the application of advanced techniques for the preparation of the samples (solid phase extraction techniques, semi-preperative high pressure chromatography, ultrasound-assisted emulsificationmicroextraction), and the identification and quantification of phenolic molecules (gas chromatography-mass spectrometry, (GC-MS), high pressure liquid chromatography-diode array detector/mass spectrometry (HPLC-DAD/MS), liquid chromatography diode array detector-electrospray time-of-flight mass spectrometry (HPLC-ESI$\mathrm{TOF} / \mathrm{MS}$ ), other hyphenated techniques and high resolution mass spectrometry (HPLC-DAD-SPE-NMR/MS, ORBITRAP platform analyzers), as well as nuclear magnetic resonance techniques) $[5,6]$ Published research work and ongoing studies are extended and their outcome may ultimately be used to integrate the results of experiments in various disciplines, including food chemistry, biochemistry, pharmacology and other biological sciences.

\section{Table olives}

Table olives that are green, turning color, or black are good sources of bioactive compounds but they have not yet been fully appreciated as a valuable functional food The phenols reported to be present in commercial samples of table olives, depending on the method of debittering, are verbascoside, hydroxytyrosol, tyrosol, luteolin, and apigenin 7-O-glycosides, and phenolic acids. Traditionally prepared table olives have been also reported that are rich sources of oleuropein. Other bioactive constituents in processed olives are maslinic and oleanolic acids, which are found in abundance in olive fruits.

\section{Other mediterranean diet constituents}

The enzyme Paraoxonase 1 (PON1) has been implicated in the prevention of cardiovascular diseases development of those conditions, especially atherosclerosis [7]. Extra virgin olive oil, the main source of fat, has been particularly effective in increasing PON1 activity. Other Mediterranean diet constituents such as nuts, fruits and vegetables, have been effective in modulating the activity of the enzyme.

Pomegranate has attracted much research interest as a source of some potent phenolic antioxidants involved in the protection of LDL and HDL from lipid oxidation The enhancement of PON1 activity by pomegranate was demonstrated in animal models and correlated to the presence of phenolics such as punicalagin, gallic acid and ellagic acid.

Nuts in the diet (walnuts, hazelnuts, almonds) may also boost memory in the elderly. A Mediterranean style diet supplemented by olive oil and nuts was found to counteract age-related cognitive decline, as indicated in a recent study [8].

\section{Dried fruits}

Traditional dried fruit such as raisins, figs, dates, apricots and many others have been a staple of Mediterranean diets for millennia. Modern research focuses on polyphenols. Dates contain quercetin, apigenin and luteolin; prunes have a very high chlorogenic acid content. Dried apricots and peaches are also important sources of carotenoids, compounds which are precursors of vitamin A and antioxidants.

A recent report conducted in Italy indicated that dried figs are excellent sources of luteolin-3,7-di-O-glucoside, luteolin 7-glucoside, apigenin-7-O-rutinoside, rutin, quercetin-3-glucoside, and cyanidin-3-O-rutinoside [9].

\section{Sesame seeds and sesame seeds paste (tahini)}

Sesame seed is a reservoir of biologically important compounds and its health promotion properties in humans are well known $[10,11]$.The bioactive components present in the seed include vitamins, phytosterols, minerals, polyunsaturated fatty acids, tocopherols and lignans such as .sesamin and sesamolin, These lignans have been shown to have a cholesterol-lowering effect in humans, to prevent high blood pressure, and to increase vitamin E supplies in animals [10,11].

\section{Strategies to Preserve and Disseminate a Healthy Diet}

\section{Health claims}

The body of science unravelling why olive bioactives such as polar phenols, phenolic acids, lignans, flavonoids triterpene acids and triterpene alcohols, squalene, alpha-tocopherol, hydroxyl-isochromans and many others is growing rapidly. Yet, the magnitude of the contribution of each active compound to the overall positive health effect cannot be easily estimated. Besides, several of the health benefits assigned to many dietary constituents are still under controversy; this can be deduced from the large number of applications rejected by the European Food Safety Authority about health claims of new foods and ingredients.

Olive oil is probably a unique case since there are two health claims for this natural product. In 2004 the Food and Drug Administration (FDA) announced the availability of a qualified health claim for monounsaturated fat from olive oil and reduced risk of coronary heart disease (http://www.fda.gov/-dms/qhcolive/htlm). These claim however, does not seem to be complete. The protective effects could be ascribed to the fatty acid composition of the oil but minor constituents, as indicated by recent studies, may be equally or even more important. The European Food Safety Authority has approved a claim for phenols in olive oil (listed in the European Community Council Regulation No. $432 / 2012$ ), concluding that a cause and effect relationship can be considered established between the phenolic and protection of LDL (low density lipoproteins) This is a claim for "Virgin olive oil with a high level of naturally present polar phenols". Authorization of the health claim aroused enthusiasm and was considered by the SMEs in 
the producing countries as a means to convey more benefits from virgin olive oil consumption to consumers. In its implementation, however, this claim seems to have some problems that derive from a lack of clarity in terminology and mainly due to the absence of a suitable and generally accepted analytical protocol for the determination of the bioactive compounds hosted under the claim [12]. If such a procedure is soon available, then analysis of a great number of samples will indicate if the required minimum amount of bioactive phenols in the health claim ( $5 \mathrm{mg}$ of "polyphenols": /20 g oil) is realistic.

\section{Quality and authentication}

Good quality is protected by proper regulations and specifications. Still authenticity and safety are not always guaranteed. Rules set by various organisations and the European Union can now be supported by DNA or chemistry based fingerprinting. Improved protocols to extract DNA and amplify DNA sequences are continuously proposed for the identification of origin, genuineness and typicality of the products, while new effective and sensitive analytical methods to detect traces of adulterants are also developed These are based on methodologies such as nuclear magnetic resonance spectroscopy, gas chromatography, high-performance liquid chromatography and hyphenated methods (HPLC-ESI-MS, MALDI-TOF MS, LC-NMR) combined with principal components or other correlation analyses $[13,14]$.

\section{The interplay of science, innovation and tradition}

Innovative food science is based on the increased awareness about the role of minor constituents. Therefore, major technological developments that aim at improving quality and preservation focus on an amelioration of the composition and retention of bioactive ingredients in the final product.

People give value to the cultural identity of food but also to the findings in the area of chemistry, nutrition and biosciences related to Mediterranean foods. Producers should identify innovative products that are also traditional. Technology and tradition, these two seemingly contradictory factors, can interplay successfully.

\section{Non cultivated vegetables}

A common feature of the Mediterranean diet, the Mediterranean aliment culture, is a high consumption of vegetable. The scientific literature concerning the Mediterranean diet and its constituents is very extensive. Still systematic ethnobotanical studies focusing on wild vegetables traditionally gathered and consumed in many areas are rather scant and sporadic [15]. Wild vegetables of the Mediterranean diet have been the subject of a series of recent phytochemical and phytopharmacological studies that indicated a reduced risk of thrombosis and related conditions due to the consumption of specific boiled plants [16]. An efficacy as anti-obesity agents was also observed [17] Still, the usual picture of the Mediterranean diet underlines mainly cultivated food plants. The wild vegetables remain yet a largely unknown section.

Strategies to preserve and evaluate further this type of food from non-cultivated plants should promote nutritional, biochemical, and other studies in the field of biosciences but also the traditional culinary heritage of a territory and sustainable gastronomy.

\section{Substitutes and imitation products}

Diet is now associated with hundreds of "healthier" products. Among them many patented preparations based on the incorporation of Mediterranean food into other food or various substitutes and imitation products promoted with the claim that they "simulate" the Mediterranean diet. These products are loosely controlled; more documentation and updated legislation is needed. Strategies are also necessary to promote consumer awareness in the countries of the Mediterranean basin but also in other countries where consumers are now seriously interested in the benefits of the real diet and the dietary patterns and lifestyle found in olive-growing areas of the Mediterranean basin.

\section{Conclusion}

The traditional Mediterranean diet is characterized by a high intake of plant products, virgin olive oil, fruits, olives, grains, bread, pulses, nuts and seeds. Moderate amounts of fish, poultry, dairy products and eggs are consumed with small amounts of red meat and wine .It emerged in the last three decades and it was introduced by the classic cross-cultural epidemiological "Seven countries study". The healthy traditional Mediterranean pattern is represented by various pyramids indicating graphically the foods to be consumed on a daily basis or weekly. Data on the association between this diet and cardiovascular disease, cancer and other chronic diseases are continuously accumulating. Mediterranean food products are now being reevaluated for the beneficial health effects they offer in relation to the bioactive compounds they contain. Olive oil constitutes a major component of the "Mediterranean diet." The chief bioactive components of olive oil include oleic acid, phenolic constituents such as the dialdehydic forms of elenolic acid linked to tyrosol and hydroxytyrosol, oleuropein and ligstroside aglycons, as well as lignans, phenolic acids, alpha-tocopherol and squalene. The European Food Safety Authority has approved a claim for phenols in olive oil recognizing that a cause and effect relationship can be considered established between the phenolics and protection of LDL (low density lipoproteins). This claim needs some clarity in the terminology of phenols and should be accompanied by standard analytical protocols for the quantitation of bioactive compounds hosted under it. Table olives are also rich sources of bioactive compounds such as oleuropein, hydroxytyrosol, lutein and apigenin glycosides and triterpenic acids. Other Mediterranean diet constituents such as nuts, pomegranate, dried fruits, sesame seeds and sesame seeds paste (tahini) have been also evaluated and found good reservoirs of bioactive compounds, mainly unsaturated fatty acids, phenolic acids, carotenoids, tocopherols, lignans and others.

Strategies to preserve and disseminate the healthy Mediterranean diet should focus on: education of the consumers who are poorly adhered to their traditional diet; implementation of the claim recently approved by EFSA for the level of biophenols in olive oil and the protection of LDL oxidation; technological improvements based on the increased awareness about the role of minor constituents of Mediterranean foods; products that are innovative but also traditional; edible non-cultivated plants and territorial culinary heritage.

\section{References}

1. Trichopoulos D, Trichopoulos A (2009) Traditional Mediterranean Diet and Health. In: Boskou D (ed) Olive Oil: Minor Constituents and Health. CRC Press, Boca Raton, FL, pp: 7-11. 
Citation: Boskou D (2016) Mediterranean Diet Food: Strategies to Preserve a Healthy Tradition. J Exp Food Chem 1: 104. doi: 10.4172/2472-0542.1000104

Page 4 of 4

2. Dermini S, Berry EM, Bach-Faig A, Behalsen R, Donini LM, et al. (2012) A dietary model constructed by scientists. In: Meditterra. CIHEAM, Sciences Po, Le Presses, Paris, pp: 71-82.

3. Estruch R, Ros E, Salas-Salvadó J, Covas MI, Corella D, et al. (2013) Primary prevention of cardiovascular disease with a Mediterranean diet. N Engl J Med 368: 1279-1290.

4. Boskou D (2015) Olive fruit, table olives, and olive oil bioactive Constituents. In: Boskou D (ed) "Olive and Olive Oil Bioactive Constituents". AOCS Press, Urbana, Illinois, pp: 1-31.

5. Kanakis P, Termentzi A, Michel T, Gikas E, Halabalaki M, et al. (2013) From olive drupes to olive oil. An HPLC-Orbitrap-based qualitative and quantitative exploration of olivekey metabolites. Planta Medica 79: 1576-1587.

6. Gómez-Caravaca AM, Lozano-Sanchez J, Contreras Gámez M, SeguraCarretero A, Taamalli A (2015) Bioactive Phenolic Compounds from Olea europaea: A Challenge for Analytical Chemistry. In: Boskou D (eds.) Olive and Olive Oil Bioactive Constituents. Elsevier, London.

7. Lou-Bonafonte JM, Gabás-Rivera C, Navarro MA, Osada J (2015) PON1 and Mediterranean Diet. Nutrients 7: 4068-4092.

8. Valls-Pedret C, Sala-Vila A, Serra-Mir M, Corella D, de la Torre R, et al. (2015) Mediterranean Diet and Age-Related Cognitive Decline. A Randomized Clinical Trial. JAMA Intern Med 175: 1094-1103.

9. Russo F, Caporaso N, Paduano A, Sacchi R (2014) Phenolic compounds in fresh and dried figs from Cilento (Italy), by considering breba crop and full crop, in comparison to Turkish and Greek dried figs. J Food Sci 79: C1278-1284.

10. Pathak N, AK Rai, Kumari R, Bhat KV (2014) Value addition in sesame: A perspective on bioactive components for enhancing utility and profitability. Pharmacogn Rev 8: 147-155.
11. Mahendra Kumar C, Singh SA (2015) Bioactive lignans from sesame (Sesamum indicum L.): evaluation of their antioxidant and antibacterial effects for food applications. J Food Sci Technol 52: 2934-2941.

12. Tsimidou M and Boskou D (2015) The health claim on "olive oil polyphenols"and the need for meaningful terminology and effective analytical protocols. Eur J Lipid Sci Technol 117: 1091-1094.

13. Perri E, Benincasa C, Muzzalupo I (2012) Olive Oil Traceability. In: Muzzalupo I (ed) Olive Germplasm - The Olive Cultivation, Table Olive and Olive Oil Industry in Italy. INTECH Rijeka, Kroatia, pp. 266-285.

14. Di Girolamo F, Masotti A, Lante I, Scapaticci M, Calvano CD, et al. (2015) A Simple and Effective Mass Spectrometric Approach to Identify the Adulteration of the Mediterranean Diet Component Extra-Virgin Olive Oil with Corn Oil. Int J Mol Sci 16: 20896-20912.

15. Biscotti N, Pieroni A (2015) The hidden Mediterranean diet: wild vegetables traditionally gathered and consumed in the Gargano area, Apulia, SE Italy. Acta Soc Bot Pol 84: 327-338.

16. Fragopoulou E, Detopoulou P, Nomikos T, Pliakis E, Panagiotakos DB, et al. (2012) Mediterranean wild plants reduce postprandial platelet aggregation in patients with metabolic syndrome. Metabolism 61: 325-334.

17. Marrelli M, Loizzo M, Nicoletti M, Confrronti F (2014) In vitro investigation of the potential health benefits of wild Mediterranean dietary plants as anti-obesity agents with a -amylase and pancreatic lipase inhibitory activities. J Sci FooD Agric 94: 2217-2224. 